\title{
Gemeinsam für eine Zukunft ohne Diabetes
}

\author{
Wie entsteht Diabetes mellitus? Wie lässt sich die Erkrankung verhindern, therapieren oder gar \\ heilen? Die Antworten auf diese Fragen kann keine Institution allein finden. Im Deutschen Zent- \\ rum für Diabetesforschung (DZD) arbeiten Spezialisten verschiedener Disziplinen zusammen, um \\ maßgeschneiderte Ansätze zur Prävention, Diagnostik und Therapie des Diabetes zu entwickeln.
}

Die Diabetesentstehung ist ein komplexer Prozess, der durch ein vielschichtiges langjähriges Zusammenspiel von Genen, Lebensstil und Umwelt zur Erkrankung führt. Nur ein integrativer Forschungsansatz, der verschiedene Disziplinen vereint, vermag das komplexe Geschehen der Diabetesentstehung zu entschlüsseln. Im DZD arbeiten Experten aus Grundlagenforschung, Epidemiologie und klinischer Anwendung deutschlandweit zusammen ( Kasten). Durch diesen translationalen Ansatz können Beobachtungen aus epidemiologischen Studien im Labor überprüft und diese Ergebnisse schneller in die klinische Anwendung gebracht werden. Ziel des DZD ist, Erkenntnisse der Diabetesforschung möglichst schnell zum Patienten zu bringen, um vorzubeugen, zu behandeln und Folgekrankheiten zu vermeiden.

In großen Bevölkerungsstudien untersuchen Forscher/-innen die Auswirkungen von Umwelt, Lebensstil und Genen auf die Entstehung des Diabetes mellitus. Neue Biomarker sollen zukünftig die Diagnose verbessern. Um personalisierte Präventions- und Therapiemaßnahmen zu entwickeln, d.h. die passende Behandlung für die richtige Patientengruppe zur richtigen Zeit, hat das DZD bundesweite klinische Multicenterstudien aufgelegt. Aktuell laufen solche zur Diabetesprävention (PLIS), zu Folgeerkrankungen (DDS), zum Schwangerschaftsdiabetes (PREG) und zur Vorbeugung von Typ-1-Diabetes (Pre-POINTearly). Mithilfe von genetischen, zellbiologischen und tierexperimentellen Techniken versuchen die Wissenschaftler sowohl mit In-vitro- als auch mit In-vivo-Versuchsansätzen die molekularen Mechanismen des Diabetes aufzuklären. So möchte man weitere Gene identifizieren, die mit Diabetes assoziiert sind, sowie epigenetische und biochemische Regulationswege aufdecken ( $\triangleright$ Abb. 1). Zudem arbeiten DZD-Experten daran, neue Wirkstoffkandidaten und Angriffspunkte für innovative Medikamente zu entdecken, zu validieren und weiterzuentwickeln. Auch wird untersucht, welche Rolle Gehirn, Leber, Muskeln, Fettgewebe und Pankreas bei der Diabetesentstehung spielen und wie Organe interagieren (organ crosstalk).

Typ-1-Diabetes ist ein weiterer Forschungsschwerpunkt. Hier gilt es, die Mechanismen zu entschlüsseln, die zur Entstehung der Autoimmunerkrankung führen, sowie Marker zu identifizieren, die eine frühe Diagnose ermöglichen, um Maßnahmen zur Prävention und Heilung von Typ-1-Diabetes zu entwickeln. Zudem arbeiten DZD-Forscher daran, die Aktivität der Betazellen zu erhalten bzw. wiederherzustellen. Um zerstörte Betazellen zu ersetzen, könnten künftig neben insulinproduzierenden Zellen menschlicher Spender auch tierische Gewebe oder Stammzellen als Quelle für Transplantate dienen.

Forschungsprojekte und -ergebnisse des DZD werden in den kommenden Ausgaben von „Info Diabetologie“ vorgestellt.

\section{Deutsches Zentrum für Diabetesforschung im Überblick}

Das DZD wurde 2009 auf Initiative des Ministeriums für Bildung und Forschung (BMBF) gegründet. Es wird zu $90 \%$ vom Bund und zu $10 \%$ von den Ländern finanziert. An den Partnerstandorten arbeiten mehr als 300 Wissenschaftler/-innen. Partner im DZD sind das Deutsche Diabetes-Zentrum in Düsseldorf, das Deutsche Institut für Ernährungsforschung in Potsdam-Rehbrücke, das Helmholtz Zentrum München - Deutsches Forschungszentrum für Gesundheit und Umwelt, das Institut für Diabetesforschung und Metabolische Erkrankungen des Helmholtz Zentrum München an der Eberhard-Karls-Universität Tübingen und das Paul-Langerhans-Institut Dresden des Helmholtz Zentrum München am Uniklinikum Carl Gustav Carus der TU Dresden. Zur Komplettierung und Stärkung der wissenschaftlichen Kompetenz integrierte das DZD Diabetesforschungsgruppen an den Universitäten in Heidelberg, Köln, Leipzig, München und Lübeck als assoziierte Partner. Weiterhin sind Wissenschaftler des ehemaligen Kompetenznetzes Diabetes mellitus Projektpartner im DZD.

Die Sprecher des Deutschen Zentrums für Diabetesforschung (DZD): Prof. Dr. Dr. h.c. Hans-Ulrich Häring, Prof. Dr. Dr. h.c. Martin Hrabě de Angelis, Prof. Dr. Dr. h.c. Michael Roden, Prof. Dr. Annette Schürmann und Prof. Dr. Dr. Michele Solimena Kontaktadressen: Ingolstädter Landstraße 1, 85764 Neuherberg; contact@dzd-ev.de; www.dzd-ev.de 